\title{
Über die Farbstoffe der Fucoideen.
}

\author{
Von \\ Harald Kylin.
}

(Aus dem medizinisch-chemischen Institut der Universität Upsala.)

(Der Redaktion zugegangen am 21. September 1912.)

Nach Cohn (1865, S. 19) gibt es bei den Fucoideen nur einen Farbstoff, den er Phäophyll nennt, und welcher für die Fucoideen dieselbe Rolle spielen soll, wie das Chlorophyll für die höheren Pflanzen. In einer Arbeit vom Jahre 1867 schreibt er (S. 44): «daß das Phäophyll ein dem Chlorophyll nahe verwandter Körper, vielleicht nur eine Modifikation desselben sei .

Rosan off (1867, S. 214) bemüht sich, nachzuweisen, daß die Fucoideen wirklich Chlorophyll enthalten, findet es aber wahrscheinlich, daß es neben diesem noch einen besonderen Farbstoff gibt. Einige Jahre später wird von Millardet (1869, S. 61) nachgewiesen, daß Benzol aus einem alkoholischen Extrakt einer Fucoidee das Chlorophyll aufnimmt, einen gelben Farbstoff aber zurückläßt, welcher Phykoxanthin genannt wird; außerdem sollen die Fucoideen einen in Wasser löslichen, braunen Farbstoff enthalten, den Millardet Phykophäin nennt.

Askenasy (1869, S. 785) erwähnt das Vorkommen zweier Farbstoffe, eines grünen und eines gelben. Sorb y (1873, S.474) unterscheidet dagegen zwei verschiedene grüne Farbstoffe, die er blaues Chlorophyll und Chlorofucin (der Farbe nach gelbgrün) nennt, und drei verschiedene gelbe Farbstoffe: orange Xanthophyll, Fukoxanthin und Lichnoxanthin; der letztere soll nur in sehr geringer Menge vorkommen, Fukoxanthin dagegen in reichlicher Menge.

Reinke (1876, S. 400) findet die Methode Sorbys zum Unterscheiden mehrerer gelber Farbstoffe nicht zuverlässig. 
Er unterscheidet nur einen gelben Farbstoff, den er Phykoxanthin nennt; dieser wäre mit dem Xanthophyll der höheren Pflanzen nicht identisch. Außerdem kämen Chlorophyll und Phykophäin vor; das Phykophäin verursache die braune Farbe der Fucoideen. - In einer Arbeit vom Jahre 1886 (S. 243) nennt er den Farbstoff der Fucoideen Phäophyll, welches seiner Ansicht nach aus einer Eiweißkomponente und einer farbigen Komponente bestehe. Beim Abtöten der Zellen würden diese Komponenten von einander getrennt. An einer anderen Stelle derselben Arbeit (S. 215) schreibt er, daß es zwei farbige Substanzen gebe, eine grüne und eine gelbe, daß es aber unentschieden bleibe, ob diese beim Abtöten der Zellen entstehen, oder ob sie schon in den lebenden Chromatophoren vorhanden seien. Hinsichtlich des Phykophäins schreibt er (S. 243): «Ich vermute in demselben einen Farbstoff, der erst durch das Eintrocknen der Fucaceen entsteht.»

Hansen (1885, S. 289) unterscheidet drei verschiedene Farbstoffe: "Chlorophyllgrün», «Chlorophyllgelb» und Phykophäin. Die beiden ersteren wären mit den grünen und gelben Farbstoffen der höheren Pflanzen identisch. - In einer Arbeit vom Jahre 1887 gibt Schütt eine eingehendere Beschreibung des Phykophäins. - Mittels der Kalimethode von Molisch hat Tammes (1900, S. 240) das Vorkommen von Carotin bei den Fucoideen nachgewiesen.

Gaidukov (1903, S. 538) leugnet das Vorkommen von Phykoxanthin bei den Fucoideen. Dieses wäre nichts anderes als ein Gemenge von Chlorophyll, Carotin und Phykophäin, oder von Carotin und Phykophäin; dieser letztere Farbstoff wird braunes Phykochrom genannt.

Nach Molisch (1905, S. 135) würden die Fucoideen Phäophyll enthalten, und dieser Farbstoff wäre «ein brauner, dem Chlorophyll nahestehender Körper». Er ist demnach derselben Meinung, die schon früher von Gohn ausgesprochen wurde. Die Chromatophoren der Fucoideen enthielten daneben Carotin und einen besonderen Stoff mit unbekannter Farbe, welcher Leukocyan genannt wird. Wird dieser Stoff mit 2\% iger Salzsäure behandelt, so entstehe ein neuer blaugefärbter Stoff, 
das Phäocyan. Das Phykophäin werde erst nach dem Tode der Alge gebildet.

Tswett (1906) unterscheidet bei den Fucoideen fünf verschiedene Chromatophorenfarbstoffe, nämlich zwei Chlorophyllmodifikationen, Chlorophyllin $\alpha$ und Chlorophyllin $\beta$ (= Chlorofucin nach Sorby) und drei gelbe Farbstoffe, Carotin, Fukoxanthin und Fukoxanthophyll. Hinsichtlich des Phykophäins schreibt er (a.a. 0., S. 236), daß es «vollständig auf eine durch alkalische Reaktion des Extraktionswassers sehr geförderte Oxydation von farblosen Chromogenen zurückzuführen ist.. Diese Behauptung habe ich durch eigene Untersuchungen schon früher bestätigt. Der Stoff, der bei seiner Oxydation Phykophäin gibt, findet sich bei den lebenden Fucoideen in den Fukosanbläschen, früher Fukosankörnchen genannt, und ist mit den Gerbstoffen nahe verwandt. Er wird Fukosan genannt (vgl. Kỳlin 1912).

Jüngst ist von Czapek (1911, S. 251) behauptet worden, daß die Fucoideen *wirklich in den lebenden Chloroplasten das gewöhnliche amorphe Chlorophyll oder Phytylchlorophyllid Willstätters enthalten». Daneben sollen sie drei gelbe Farbstoffe enthalten: Carotin, Xanthophyll und Fukoxanthin.

\section{Chlorophyll.}

Ob Chlorophyll in den Chromatophoren der Fucoideen nativ vorkommt oder nicht, ist eine Frage, die in der Literatur mehrmals in verschiedener Weise beantwortet worden ist. Molisch (1905, S. 135) vertritt die Meinung, daß die lebenden Fucoideen kein Chlorophyll enthalten, sondern einen braunen dem Chlorophyll nahestehenden Körper, das Phäophyll, welches beim Eintauchen in siedendes Wasser in Chlorophyll umgewandelt würde. Tswett (1906) behauptet dagegen, daß die lebenden Fucoideen Chlorophyll enthalten. Jüngst ist die letztere Meinung von Czapek (1911, S. 250) bestätigt worden, und auf Grund eigener Untersuchungen muß ich mich entschieden dieser Meinung anschließen.

Nach den Untersuchungen von Willstätter enthält das Chlorophyll Magnesium. Willstätter (1906, S. 62) hat auch

Hoppe-Seyler's Zeitschrift f. physiol. Chemie. LXXXII. 
nachgewiesen, daß das Chlorophyll aus einer Fucus-Art Magnesium enthielt, und während meiner Untersuchungen habe ich dargetan, daß aus Ascophyllum nodosum extrahiertes Chlorophyll magnesiumhaltig war. Das Chlorophyll wurde nach der von Willstätter (a. a. 0., S. 74) beschriebenen Phosphatmethode gereinigt.

Es gibt bei den höheren Pflanzen zwei verschiedene Chlorophyllmodifikationen, eine blaugrüne und eine gelbgrüne. Diese Modifikationen werden von Willstätter Chlorophyll a und Chlorophyll b genannt, von Marchlewsky dagegen Neochlorophyll und Allochlorophyll, von Tswett Chlorophyllin $\alpha$ und Chlorophyllin $\beta$. Bei den Fucoideen solle nach Tswett (1906, S. 239) die erstere dieser Modifikationen vorkommen, die letztere dagegen nicht, anstatt dieser käme aber eine andere gelbgrüne Modifikation vor, die Chlorophyllin $\curlyvee$ genannt wird (= Chlorofucin nach Sorby, 1873, S. 454). Dieser Frage habe ich indessen keine genauere Erörterung gewidmet (in Willstätters Laboratorium soll man sich gegenwärtig mit einer Untersuchung der Fucoideenchlorophylle beschäftigen).

\section{Carotin.}

Mittels Molischs Kalimethode (Molisch 1896, S. 19) hat Tammes das Vorkommen von Carotin bei folgenden Fucoid'een nachgewiesen: Fucus vesiculosus, F. serratus, Laminària saccharina, L. digitata, Chorda filum und Ascophyllum nodosum. Nach derselben Methode habe ich eine größere Zahl Fucoideen untersucht, und es gelang mir immer sehr gut, Carotin riachzuweisen.

Um Carotin makrochemisch nachzuweisen, wurde an der Luft -getrocknetes, sehr fein pulverisiertes Material von Ascophyllum nodosum mit Petroläther extrahiert. Das Extrakt. wurde dann hauptsächlich nach der Methode von Willstätter (1907, S. 12) gereinigt, und die schließlich erhaltene Lösung von Carotin in Schwefelkohlenstoff wurde mit Alkohol versetzt. Nach etwa einer Stunde waren prachtvolle Carotinkrystalle gebildet worden, die der Form nach mit der von Willstätter (a. a. 0., S. 17) gegebenen Figur sehr gut übereinstimmten. 


\section{Xanthophyll.}

Um Xanthophyll bei den Fucoideen nachzuweisen, bediente ich mich der von Willstätter (1907, S. 13) beschriebenen Methode zur Gewinnung von Xanthophyll aus Blättern. Ein alkoholisches Extrakt wurde demnach mit alkoholischem Alkali versetzt, um das Chlorophyll zu verseifen, und danach mit Äther ausgeschüttelt. Die ätherische Lösung wurde mit Wasser gewaschen, mit Natriumsulfat getrocknet, im Vakuum stark eingeengt und schließlich mit Petroläther versetzt, wobei das Xanthophyll als ein pulvriger, rötlichgelber Niederschlag gefällt wurde. Da ich nur mit verhältnismäßig geringeren Mengen von Ausgangsmaterial arbeitete, war die erhaltene Xanthophyllmenge zu gering, um zum Zwecke der Reindarstellung weiter bearbeitet zu werden. - In der oben beschriebenen Weise habe ich das Vorkommen von Xanthophyll bei Ascophyllum nodosum, Fucus vesiculosus, F. serratus, Halidrys. siliquosa, Laminaria digitata und Pylaiella litoralis bewiesen.

In folgender Weise habe ich das Fucoideenxanthophyll zum Krystallisieren gebracht. An der Luft getrocknetes, fein pulverisiertes Material wird mit Benzol extrahiert, und das Extrakt mit Alkohol ausgeschüttelt. Der größte Teil des Xanthophylls und Phykoxanthins geht dabei in den Alkohol über, Carotin und Chlorophyll (nur in geringer Menge extrahiert) bleiben dagegen größtenteils im Benzol. Die alkoholische Lösung wird dann bei Zimmertemperatur allmählich verdampft, und man kann dabei das Xanthophyll in der Form von orangeroten, unregelmäßig tafelförmigen. Krystallen erhalten; das Phykoxanthin wird in kleinen Tröpfchen niedergeschlagen. Nach dem Auskrystallisieren des Xanthophylls wird die Mutterlauge vorsichtig abdekantiert, und der Rückstand bei Zimmertemperatur im Vakuum getrocknet. Der getrocknete Rückstand wird mit Petroläther behandelt. Das Phykoxanthin (nebst den Verunreinigungen von Carotin und Chlorophyll) wird dabei gelöst, und der orangerote Rückstand von Xanthophyll läßt sich gut abfiltrieren (ein Teil bleibt als ein orangeroter Belag auf 
dem Boden des Gefäßes zurück; die Beimischungen von Phykoxanthin vollkommen $\mathrm{zu}$ entfernen, ist schwierig $\left.{ }^{1}\right)$ ). Nach dem Abfiltrieren kann man das Xanthophyll in etwas Alkohol leicht lösen. - Auf diese Weise habe ich das Xanthophyll aus Ascophyllum nodosum, Fucus vesiculosus, Laminaria digitata und Pylaiella litoralis zum Krystallisieren gebracht.

Bei den Fucoideen gibt es demnach einen krystallisierenden, in Petroläther unlöslichen, in Alkohol aber leicht löslichen gelben Farbstoff, der mit dem von Willstätter untersuchten Xanthophyll der höheren Pflanzen identisch sein dürfte. Von konzentrierter Schwefelsäure wird er blau gefärbt, von stärkerer alkoholischer Salzsäure bei kurzem Erwärmen zuerst grün, dann blaugrün bis blau, in ähnlicher Weise wie das Xanthophyll der höheren Pflanzen.

Dieser Farbstoff ist zuerst von Tswett (1906, S. 240) bei den Fucoideen nachgewiesen worden, und wird von ihm als Fukoxanthophyll bezeichnet.

\section{Phykoxanthin.}

Der Name Phykoxanthin wird zum erstenmal von Nägeli (1849, S. 7) benutzt. Er behauptet, es gebe bei den Cyanophyceen einen besonderen Farbstoff, den er Phykochrom nennt, und welcher in zwei Modifikationen vorkommen soll, einer blaugrünen, Phykocyan, und einer orangefarbigen, Phykoxanthin. Kraus und Millardet (1868, S. 26) bezeichnen mit dem Namen Phykoxanthin denjenigen gelben Farbstoff der Cyanophyceen und Diatomeen, der im Alkohol zurückbleibt, wenn ein Alkoholextrakt dieser Algen mit Benzol ausgeschüttelt wird, und mit demselben Namen bezeichnet auch Millardet (1869,

1) In meinem Aufsatz «Über die grünen und gelben Farbstoffe der Florideen wurde behauptet (S. 118), daß eine aus Geramium rabrum dargestellte Xanthophyllösung beim Kochen nach Zusatz von etwas Eisessig grün wird, und daß sie bei Behandlung mit Alkali in irgend einer Weise verändert wird, so daß die Farbe nach Zusatz von einer Säure bis zu saurer Reaktion augenblicklich grün wird. Diese Angaben beruhen darauf, daß meine Xanthophyllösungen mit Phykoxanthin verunreinigt waren. Eine reine Xanthophyllösung gibt diese Reaktionen nicht. 
S. 60) denjenigen gelben Farbstoff der Fucoideen, der im Alkohol zurückbleibt, wenn ein Alkoholextrakt dieser Algen mit Benzol ausgeschüttelt wird. Sorby $(1873$, S. 461) verändert den Namen Phykoxanthin zu Fukoxanthin und will mit diesem Namen den besonderen gelben Farbstoff der Fucoideen bezeichnen. In der späteren Literatur wird in der Regel der letztere Name benutzt; in der folgenden Darstellung soll aber der ältere Name Phykoxanthin verwendet werden. - Sowohl die Fukoxanthinlösung nach Sorby wie die Phykoxanthinlösungen der früheren Forscher waren mit Xanthophyll vermischt.

Das Phykoxanthin steht ganz gewiß dem Xanthophyll sehr nahe, unterscheidet sich aber von diesem dadurch, daß es in Petroläther löslich ist; Xanthophyll ist im Petroläther unlöslich. Wird eine Lösung von Phykoxanthin in Petroläther mit $80 \%$ igem Alkohol ausgeschüttelt, geht der Farbstoff so gut wie vollständig in den Alkohol über. Carotin würde bei einer solchen Ausschüttelung im Petroläther bleiben.

Eine verdünnte Lösung von Phykoxanthin in Alkohol, Benzol oder Petroläther ist der Farbe nach nicht von einer verdünnten Xanthophyll- oder Carotinlösung zu unterscheiden. Eine konzentrierte Phykoxanthinlösung wird aber orangebraun, eine konzentrierte Lösung von Xanthophyll oder Carotin dagegen orangerot.

Verdünnte Salzsäure oder Schwefelsäure färben eine Phykoxanthinlösung bei höherer Temperatur sehr schnell, bei Zimmertemperatur etwas langsamer zuerst grün, dann blaugrün bis schön blau. Bei Alkalisieren wird die Farbe wieder gelb. Eine Xanthophyllösung wird auch von Salzsäure oder Schwefelsäure grünblau gefärbt, die Reaktion vollzieht sich aber viel langsamer und man muß eine etwas stärkere Säure verwenden. (Molischs Leukocyan ist eben Phykoxanthin, und sein Phäocyan ist nichts anderes als durch Säure blau gefärbtes Phykoxanthin).

Eine Phykoxanthinlösung wird auch durch konzentrierte Essigsäure grün bis blaugrün gefärbt, die Reaktion vollzieht sich aber viel langsamer, als bei Verwendung von Mineralsäuren. Oxalsäure wirkt wie Essigsäure, aber etwas kräftiger. 
Xanthophyllösungen werden von Essigsäure oder Oxalsäure nicht grün gefärbt. Xanthophyllkrystalle lösen sich in Eisessig, verändern jedoch die Farbe nicht einmal beim Kochen.

Bei Behandlung mit verdünnter Kali- oder Natronlauge wird eine Phykoxanthinlösung beim Kochen schnell, bei Zimmertemperatur nach einigen Minuten in irgend einer Weise verändert, so daß die Farbe nach Zusatz von einer Säure bis zu saurer Reaktion augenblicklich grün wird. Bei Alkalisieren wird die Farbe wieder gelb. Eine Xanthophyllösung wird von Alkali nicht verändert. Schon früher ist von Tswett (1906, S. 240) nachgewiesen worden, daß eine Phykoxanthinlösung von Alkalien angegriffen wird, und daß sie sich dabei spektroskopisch verändert.

Zur Gewinnung von Phykoxanthinlösungen extrahiert man an der Luft getrocknetes, fein pulverisiertes Material einer Fucoidee mit Petroläther. Das Extrakt wird mit Alkohol ausgeschüttelt, wobei Carotin und Chlorophyll größtenteils im Petroläther zurückbleiben, das Phykoxanthin in die Alkoholschicht übergeht. Um die Beimischungen von Carotin und Chlorophyll $\mathrm{zu}$ entfernen, wird dann die Alkoholschicht einigemal mit Petroläther ausgeschültelt. Um die Reinigung weiter zu treiben, wird die alkoholische Lösung mit Äther ausgeschüttelt, die ätherische Lösung mit Wasser gewaschen, mit Natriumsulfat getrocknet und im Vakuum bis zur Trockenheit eingedampft. Der Rückstand wird dann mit Petroläther behandelt, wobei das Phykoxanthin gelöst wird, und der Farbstoff kann dann wieder in Alkohol hinübergeschüttelt werden. Auf diese Weise kann man die Beimischungen von Chlorophyll und Carotin entfernen, nicht aber die farblosen Beimischungen (reine Phykoxanthinlösungen habe ich nicht dargestellt). Das Xanthophyll ist in Petroläther nicht löslich und würde demnach nach dieser Methode nicht extrahiert werden. Es zeigte sich aber, daß der oben erwähnte Rückstand nach dem Eindampfen der ätherischen Lösung sich nicht vollkommen im Petroläther löste, sondern etwas Xanthophyll hinterließ. Vielleicht erhöhen die farblosen Beimischungen die Löslichkeit des Xanthophylls in Petroläther. 
Auch in folgender Weise lassen sich Phykoxanthinlösungen gut darstellen. Das fein pulverisierte Material wird mit 75\% igem Alkohol extrahiert. Chlorophyll und Carotin lösen sich dabei nur in geringeren Mengen, Phykoxanthin und Xanthophyll dagegen sehr reichlich. Das alkoholische Extrakt wird mit Petroläther ausgeschüttelt, um Carotin und Chlorophyll zu entfernen; Phykoxanthin und Xanthophyll werden dann in Äther hinübergeschüttelt. Die ätherische Lösung wird dann mit Wasser gewaschen, mit Natriumsulfat getrocknet, im Vakuum bis zur Trockenheit eingedampft. Wird der so erhaltene Rückstand mit Petroläther behandelt, löst sich das Phykoxanthin, das Xanthophyll bleibt aber ungelöst zurück.

\section{Literaturverzeichnis.}

Askenasy, E., Beiträge zur Kenntnis der Gattung Ectocarpus. Bot. Zeitung, Bd. 27, 1869.

Cohn, F., Über einige Algen von Helgoland. Beiträge zur näheren Kenntnis und Verbreitung der Algen, herausgegeben von Dr. L. Rabenhorst, Heft 2, Leipzig 1865.

- Beiträge zur Physiologie der Phykochromaceen und Florideen. Archiv für mikroskopische Anatomie, herausgegeben von $\mathrm{Max}$ Schultze, Bd. 3, 1867.

Czapek, Fr., Über die Farbstoffe der Fukazeen. Lotos, Bd. 59, Prag 1911. Gaidukov, N., Über den braunen Algenfarbstoff. Berichte d. deutsch. bot. Ges., Bd. 21, 1903.

Hansen, A., Chlorophyllgrün der Fucaceen. Arb. d. bot. Inst. Würzburg, Bd. 3, 1885.

Kraus, G. et Millardet, A., Études sur la matière colorante des Phycochromacées et des Diatomées. Mém. de la soc. des sc. nat. de Strasbourg, Bd. 6, 1868.

$\dddot{K}$ ylin, H., Über die grünen und gelben Farbstoffe der Florideen. Diese Zeitschrift, Bd. 74, Straßburg 1911.

- - Über die Inhaltskörper der Fucoideen. Arkiv för Botanik, Utgifvet af K. V. Akadem. i Stockholm, Bd. 11, Nr. 5, 1912.

Millardet, A., Sur la nature du pigment des Fucoidées. Annales des sc. nat., Botanique, S. 5, T. 10, 1869.

Molisch, H., Die Krystallisation und der Nachweis des Xanthophylls (Carotins) im Blatte. Berichte d. deutsch. bot. Ges., Bd. 14, 1896.

- - Über den braunen Farbstoff der Phaeophyceen und Diatomeen. Bot. Zeitung, Bd. 63, 1905.

$\mathrm{Nägeli,} \mathrm{C.,} \mathrm{Gattungen} \mathrm{einzelliger} \mathrm{Algen,} 1849$. 
Reinke, J., Beitrag zur Kenntnis des Phykoxanthins. Pringsheims Jahrbücher, Bd. 10, 1876.

- - Photometrische Untersuchungen über die Absorption des Lichtes in den Assimilationsorganen. Bot. Zeitung, Bd. 44, 1886.

Rosanoff, S., Observations sur les fonctions et les propriétés des pigments de diverses Algues. Mém. de la Soc. imp. des sc. nat. de Cherbourg, Bd. 13, 1867.

Schütt, Fr., Über das Phykophaein. Berichte d. deutsch. bot. Ges., Bd. 5, 1887.

Sorby, H. C., On comparative vegetable Chromatology. Proceed. Roy. Soc., Bd. 21, London 1873.

Tammes, T., Über die Verbreitung des Carotins im Pflanzenreiche. Flora, 87, 1900.

Tswett, M., Zur Kenntnis der Phaeophyceenfarbstoffe. Berichte d. deutsch. bot. Ges., Bd. 24, 1906.

Willstätter, R., Zur Kenntnis der Zusammensetzung des Chlorophylls. Liebigs Annalen der Chemie, Bd. 350, 1906.

- - und Mieg, W., Über die gelben Begleiter des Chlorophylls. Ebenda, Bd. 355, 1907. 